\title{
Metodología 5S, alternativa viable en la mejora de procesos de la industria alimentaria
}

\section{S Methodology, viable alternative in the improvement of food industry processes}

\author{
iD 'Carlos Augusto Salazar Sandoval iD 'Harold Pawel Johao Ore Quiroz iD 'Brenda Jackeline Benavides \\ Alvarado iD 'Yenifer Alexandra Delgado Calderón iD ${ }^{2}$ Lucia Pantoja-Tirado
}

Universidad Nacional de Frontera

Universidad Nacional Autónoma de Tayacaja Daniel Hernández Morillo²

\section{Resumen}

Excelencia en base a calidad es prioritario en la empresa moderna. La filosofía Kaizen, aplicada por la Toyota, es el caso de éxito mundial más emblemático y demuestra que con esta metodología es posible elevar los estándares de productividad en las empresas. En Latinoamérica y sobre todo en el Perú, aún estas técnicas son poco conocidas. El objetivo de esta investigación fue divulgar la efectividad de la estrategia base para el Kaizen: la metodología de las 5S. Partiendo de la premisa Kaizen "que todo cuanto existe se puede mejorar", las 55 son fundamentales en los procesos de mejora continua representando una alternativa en las empresas, para lograr efectos considerablemente positivos; sin embargo, por muy fácil y de bajo costo que parezca su implementación, esta demanda de mucho compromiso, desde la alta gerencia hasta el empleado del último orden jerárquico de la empresa.

Palabras clave: 5S, eficiencia, calidad, Kaizen, productividad, seguridad.

Recibido: Junio 2020

Aceptado: Setiembre 2020

\section{Abstract}

Excellence based on quality is a priority in the modern company. The Kaizen philosophy, applied by Toyota, is the most emblematic world success story and shows that with this methodology it is possible to raise productivity standards in companies. In Latin America and especially in Peru, even these techniques are little known. The objective of this research was to disclose the effectiveness of the base strategy for Kaizen: the $5 \mathrm{~S}$ methodology. Starting from the Kaizen premise "that everything that exists can be improved", the $5 \mathrm{~S}$ are fundamental in continuous improvement processes, representing an alternative in companies, to achieve considerably positive effects; However, no matter how easy and inexpensive its implementation may seem, this demands a lot of commitment, from his demands a lot of commitment, from the top management to the employee of the last hierarchical order of the company of the company.

Keywords: 5S, efficiency, quality, Kaizen, productivity, safety. 


\section{INTRODUCCIÓN}

Las empresas hoy en día buscan la excelencia en todas sus actividades comerciales; procesos más seguros, con menos pérdidas y que generen más ganancias, es lo que mueve a las grandes empresas a reorganizarse y buscar caminos que los conducen a ella. Las empresas transnacionales o de orden global, son conscientes de ello, por lo que hoy en día aplican estrategias como el Kaizen o el Lean Manufacturing y todas sus herramientas entre ellas, las $5 S$ muy poco conocidas aún en nuestro medio.

Karla S. Almonte (2011), en su artículo Kaizen y las 5S, sostiene que la palabra Kaizen es muy nombrada hoy en día en varios idiomas, fundamentando su popularidad debido al éxito asociado a la filosofía del sistema de producción del Toyota Japonés de los años 50. "Hoy mejor que ayer, mañana mejor que hoy", afirma, es la base de la milenaria filosofía Kaizen. Bajo este concepto, siempre es posible optimizar; y en eso, el tiempo ha demostrado que los japoneses no se equivocaron, ya que Japón es un país altamente industrializado, tiene las mejores economías del planeta y es altamente competitivo. En la cultura japonesa es una regla que ningún día debe pasar sin una cierta mejora.

Después de la segunda guerra mundial; con la ocupación de Japón por fuerzas militares estadounidenses llegaron con ellos expertos en métodos estadísticos de Control de calidad de procesos, para los programas de entrenamiento denominados TWI (Training Within Industry) los mismos que brindaban servicios de consultoría a las industrias relacionadas con la Guerra; fueron dirigidos por W. Edwards Deming y Joseph M. Juran y finalmente se orientaron a instruir a la industria civil japonesa, en métodos de trabajo como el control estadístico de procesos y otras técnicas. La aplicación de estas estrategias aplicadas en la industria japonesa llevó al país a estar entre las principales economías del mundo.
Definitivamente la necesidad de salir de la crisis económica, sumada a la filosofía y disciplina típica de la cultura japonesa; además de estas herramientas estadísticas llevadas por los norteamericanos al Japón de los años 50, crearon esta nueva filosofía aplicada a los negocios que ha traído éxito y desarrollo a muchas economías empresariales.

Las $5 \mathrm{~S}$ se manifiestan hoy en día como el primer paso para la excelencia en la calidad aplicada no solo a las industrias, sino también a cualquier campo del ámbito empresarial. Definitivamente, las empresas hoy en día mediante políticas orientadas a la satisfacción del cliente muy conocedoras de su entorno buscan tener mayor rapidez de respuesta en la atención de las necesidades de los clientes; competitividad frente a la economía global empresarial, más flexibilidad y aumento en la capacidad instalada y capacidad de producción; y sobre todo cumplir los estándares de calidad e inocuidad en toda la cadena productiva y así asegurar alta demanda de sus productos.

Además de lo mencionado, a nivel de mejorar la calidad de los procesos y del producto también la seguridad y salud ocupacional de sus colaboradores, el ambiente de trabajo y el clima laboral, la comunicación interna; desarroIla la creatividad y permite el crecimiento de los colaboradores, mejora y desarrolla su autoestima e incrementa los factores positivos en la cultura organizacional de las empresas.

La estrategia de las 5S implica alto nivel de involucramiento de la gerencia general de la empresa, mucha capacitación para todos sus miembros desde el gerente hasta el servidor del ultimo nivel jerárquico. Carvalho de Souza et al., (2018) manifiestan que la implementación y mantenimiento del programa $5 \mathrm{~S}$ no es una tarea sencilla, debido a que está directamente relacionado con el comportamiento y la cultura de las personas, hecho por el cual muchas empresas no logran los resultados esperados al implementar esta estrategia. 
Las personas traen sus propios estilos de vida desde sus hogares. Bajo la percepción de algunas es muy común que un área desordenada parezca que no lo es; por lo que al llegar a un centro de labores donde estas percepciones son diferentes y sobre todo una exigencia en el ámbito laboral, se generan conflictos. Pedir que las personas cambien sus percepciones es sumamente difícil, debido a que esa cultura propia está arraigada desde el hogar, Según Chiavenato (2008), cambiar significa pasar de un estado a otro diferente. Es la transición de una situación a otra. El cambio implica transformación, interrupción, perturbación o ruptura, dependiendo de su intensidad. Para una persona tradicional en nuestra cultura este proceso de adaptación y de aprendizaje de nuevos estilos de vida, puede resultar un trabajo muy arduo y demandar de mucho tiempo; este proceso de cambio pasa por tres etapas: descongelación, cambio y recongelación.

Hablar de descongelación es hablar de desaprender, es decir, empezar a dejar de lado percepciones y conceptos culturales arraigados en nuestra cultura e iniciar a pensar que se puede cambiar para mejorar. Es romper paradigmas, de hábitos que probablemente se asumen que nos hacen una vida confortable y sin complicaciones y empezar a trazarnos metas más complejas, retos basados en disciplina y en valores, que le podemos dar valor agregado a nuestras acciones. La etapa de descongelamiento es muy importante ya que, si no logramos desaprender, es difícil aprender nuevos conceptos, consecuentemente tener nuevas percepciones.

Terminada la etapa de descongelamiento viene la etapa de cambio, de aprendizaje, donde se empiezan a adquirir nuevas percepciones, estilos. Es esta la parte crucial del proceso de implementación de las 5S. La etapa de aprendizaje pasa primero por una etapa de diag- nóstico. Una auditoria previa para conocer la realidad y el estado actual de la gestión, para conocer las ventajas y desventajas precedentes a la implementación y sobre todo el grado de impacto que este va a generar en los colaboradores. El objetivo final de la implementación se encuentra en esta etapa y para ello son utilizadas diferentes técnicas o metodologías muchas de ellas herramientas de calidad asociadas al mismo, para planificar, analizar, proponer mejoras. y controlar la eficiencia del programa 5S.

Muchas de las herramientas usadas forman parte de la estrategia del Sig Sigma; Carvalho et al., (2018) en su artículo Implementación del programa 55 a través de la metodología DMAIC (cuyo nombre es el acrónimo en inglés de las cinco etapas para este proceso de mejora: Definir, Medir, Analizar, Evaluar y Controlar), proponen el uso de esta herramienta, la cual permite identificar, cuantificar y reducir la variabilidad de un proceso. Otra de las propuestas de Carvalho et al., (2018) es el uso de la Matriz GUT, herramienta que se utiliza para analizar situaciones y priorizar estrategias, toma de decisiones y resolución de problemas en el ámbito gerencial. Analiza un problema o una situación particular, determina las causas raíz de los mismos y a través de ello se puede cuantificar y clasificar los problemas (Gomes, 2006), además de facilitar la priorización de acciones según esta clasificación.

Los tres atributos principales de clasificación de problemas analizados en la matriz de GUT son Gravedad, Urgencia y Tendencia, para determinar la prioridad de atención a un problema reciben valores de acuerdo a esta del 1 al 5, según la experiencia y la percepción de quién está evaluando; el producto de estos tres atributos indica la prioridad de atención de un problema, que tendrá en cuenta la mayor prioridad a aquel que arroje el valor más elevado, según la siguiente tabla 1. 


\section{Tabla 1}

\section{Valoración de la matriz de GUT son Gravedad, Urgencia y Tendencia}

\begin{tabular}{cccc}
\hline VALORACIÓN & GRAVEDAD & URGENCIA & TENDENCIA \\
\hline 5 & $\begin{array}{c}\text { Extremadamente } \\
\text { severo }\end{array}$ & Acción inmediata & $\begin{array}{c}\text { Sin acción se volverá más } \\
\text { serio }\end{array}$ \\
4 & Muy Serio & Con cierta urgencia & $\begin{array}{c}\text { Empeoramiento a corto } \\
\text { plazo }\end{array}$ \\
3 & Grave & $\begin{array}{c}\text { Tan pronto como } \\
\text { sea posible }\end{array}$ & $\begin{array}{c}\text { Empeoramiento a medio } \\
\text { plazo }\end{array}$ \\
& Poco serio & Puede esperar & $\begin{array}{c}\text { Empeoramiento a largo } \\
\text { plazo }\end{array}$ \\
1 & Ingrávido & Puede esperar mas & No empeora \\
\hline
\end{tabular}

Determinar los problemas presentes en un proceso, su nivel de importancia y prioridad en su atención, es fundamental para su implementación. Durante la etapa de cambio, el uso de múltiples herramientas de gestión es fundamental para la pronta implementación de las 5S; herramientas como listas de verificación, entrevistas, paretos, gestión visual, Iluvia de ideas (brainstorming), diagramas causa efecto, diagramas de radar, etc.

Durante la etapa de cambio. la cual implica empezar a aprender nuevos conceptos, adoptar nuevos hábitos y costumbres. La implementación de las $5 \mathrm{~S}$ se soporta en el trabajo en equipo a alto nivel, ya que involucran a todos los colaboradores de la empresa e incluye a dueños o directores de la empresa. El compromiso con adoptar la filosofía de las $5 S$ parte desde el propio puesto de trabajo, cada trabajador contribuye y aporta su conocimiento y experiencia. Es así. que el proceso de mejora continua se hace una tarea de todos. Terminada la etapa de aprendizaje se avanza a la etapa de congelamiento, donde se establecieron nuevos hábitos, los procesos se mecanizan y hasta se vuelven rutinarios. Esta etapa se debe mantener, lo que no implica que pueda cambiarse ya que, como todo proceso de mejora continua, está sujeto al cambio.

La constante supervisión y control finalmente indicará el momento adecuado para volver al punto inicial de desaprender lo ya aprendido o a descongelar, a aprender y a volver a congelar. Los procesos productivos son cambiantes por lo que nuestros sistemas de gestión deben estar sometidos al cambio. Como lo menciona la metodología Kaizen "todo cuanto existe se puede mejorar", pues un correcto manejo e implementación de las 55 se puede optimizar, es por ello que está sometida a un ciclo de cambio.

\section{DEFINICIONES DE LAS 5S}

Hablar de la metodología 5S es hablar de cinco pasos camino al éxito. Son las iniciales de cinco palabras japonesas, que en la década de los 50 dio el éxito que hasta hoy conserva la muy conocida empresa de autos Toyota y que además marcó un hito en el desarrollo empresarial de la gran mayoría de empresa exitosas de orden mundial. Las empresas hoy en día, para desarrollar altos niveles de competitividad necesitan utilizar métodos y herramientas que les ayuden a elevar los niveles de calidad en sus procesos y en sus niveles de producción con el menor costo posible y con la consigna de superar plenamente las expectativas de sus clientes.

$5 S$ denota cinco operaciones básicas y elementales que toda compañía camino a la excelencia debe seguir: Seiri - Seito, Seiso Seiketsu - Shitsuke que en el idioma español se traduce como Clasificamos - Ordenamos - Limpiamos - Estandarizamos -Disciplina- 
mos; y por sus iniciales japonesas dan origen al nombre de esta metodología.

\section{SEIRI}

Significa Seleccionar, clasificar y se refiere a que en todas las áreas del trabajo se debe separar elementos innecesarios de los que son necesarios y posteriormente descartar lo innecesario. El acumular cosas es muy propio de nuestra cultura; para todos nosotros es muy común que en nuestros hogares se guardan cosas inútiles pensando en algún momento darle utilidad; sin embargo, esto nunca se da, lo que genera acumulaciones innecesarias que posteriormente se vuelven molestosas y difíciles de tolerar.

Matheos (2010) clasifica los objetos innecesarios de su lugar de trabajo mediante la selección de los objetos, según su utilidad, con el fin de eliminar obstáculos, reducir costos, disminuir existencias y utilizar eficientemente los recursos de la empresa.

El propósito de la selección es retirar del ambiente de trabajo todos los elementos innecesarios para las operaciones cotidianas, manteniendo así cerca los elementos necesarios que facilitan la labor del trabajo ya que de esta manera se eliminan obstáculos que pueden conllevar a paradas innecesarias de las actividades del trabajo, se previenen operaciones erróneas y se reducen focos de infección o contaminación cruzada. El trabajo de los operadores y operarios o del responsable del proceso es responsable de la clasificación, debido a que ellos son los que conocen su área de trabajo y saben lo que exactamente necesitan para un buen desempeño de su labor.

Para establecer esta "S" basta con hacerse dos preguntas simples: ¿está aprovechado el espacio en su organización o empresa al máximo, de manera eficiente y racional? ¿todo el mundo tiene el material - documentación y herramientas - necesario para desarrollar su labor? Fundación Vasca para la Excelencia Euskalit. (SF)

\section{SEITON}

Cruz (2010). "Un lugar para cada cosa y cada cosa en su lugar" es la frase que resume en toda su magnitud esta estrategia. Seiton significa Ordenar u Organizar; colocar lo necesario en lugares fácilmente accesibles, según la frecuencia y secuencia de uso, es decir, consiste en establecer el modo en que deben ubicarse e identificarse los materiales necesarios, de manera que sea fácil y rápido encontrarlos, utilizarlos y reponerlos.

Mateos (2010), refiere que ordenar los objetos necesarios en su lugar de trabajo mediante el uso de estantes, bodegas bien rotuladas con el fin de darles la ubicación adecuada para mejorar la productividad, resume el significado de la estrategia Seiton.

La implementación de esta estrategia tiene como propósito reducir los tiempos de búsqueda, reducir movimientos innecesarios dentro del área de labores, evitar confusiones peligrosas, permitir la ubicación de materiales, documentos de forma rápida; un ambiente ordenado da la impresión de que todo marcha bien, que las cosas se hacen bien y por lo tanto el producto final es de buena calidad; mejora el manejo de existencias y la coordinación para la ejecución de las tareas.

Una pregunta sencilla que nos ayuda a reconocer si estamos cumpliendo con esta estrategia es: ¿encuentra cualquier herramienta o documento en menos de 30 segundos y sin necesidad de desplazarse de su puesto de trabajo o de preguntar a otros? Fundación Vasca para la Excelencia - Euskalit (SF).

Ordenar es establecer la manera en que los materiales necesarios deben ubicarse e identificarse para que "cualquiera" pueda encontrarlos, usarlos y reponerlos de forma rápida y fácil

\section{SEISO}

Limpieza no es limpiar, sino evitar que las áreas de trabajo se ensucien. Seiso significa limpieza. TGP Hoshin (sf), manifiesta que, en 
una empresa industrial, la limpieza se relaciona estrechamente con la habilidad de producir productos de calidad. La limpieza incluye también ahorrar tareas, encontrando modos de evitar la suciedad, el polvo y toda la suciedad que pueda acumularse en el lugar de trabajo.

Mateos (2010) dice que limpiar e inspeccionar equipos, instalaciones, superficies de contacto mediante cronograma de actividades bien definido con el fin de evitar contaminaciones, alargar la vida útil de los equipos y hacer de su trabajo un lugar agradable es el objetivo de la limpieza.

Mantener limpio el puesto de trabajo asegurando que todo se encuentra siempre en perfecto estado de uso, identificar y eliminar las fuentes de suciedad, los lugares difíciles de limpiar, las piezas deterioradas o dañadas, implica establecer y aplicar procedimientos de limpieza, como ya se ha manifestado al inicio, la idea es actuar con un enfoque preventivo: No se trata de limpiar cada vez más; sino de evitar que nuestro lugar de trabajo y nuestras maquinas se ensucien.

Para saber si es que en realidad estamos cumpliendo con esta estrategia basta con hacernos estas preguntas básicas: ¿se ve obligado a dedicar alguna jornada a limpiar en vez de trabajar normalmente? ¿ha habido en su empresa averías en la maquinaria por falta de limpieza? Fundación Vasca para la Excelencia - Euskalit (sf).

Una planta limpia es una planta que además de ser un lugar agradable para trabajar; se evita problemas por contaminación cruzada, se prolonga la vida útil de los equipos, la planta se convierte en una planta segura para trabajar; la limpieza es la base del mantenimiento preventivo ya que mediante esta podemos detectar a tiempo muchos desperfectos de las máquinas antes de su puesta en marcha. La limpieza es un factor común de los procesos altamente productivos, y es una tarea que exige constancia y participación de todos; el trabajo en equipo es fundamental.

\section{SEIKETSU}

Estandarizar o mantener es el significado de esta estrategia e implica la aplicación de las (3S) anteriores, de tal manera que estas se conviertan en una rutina o acto reflejo. Esto se logra mediante el levantamiento de documentación y definición de responsabilidades, para que continúen con el tiempo, dando los beneficios que la empresa requiere para su mejoramiento continuo.

Estandarizar implica que no importa quien está al mando o quien realiza una actividad, los procesos se realizan en función a actividades establecidas no a personas indicadas. De esta manera se pueden hacer dos preguntas básicas para detectar si estamos cumpliendo con esta estrategia: ¿puede alguien ajeno a un área o sección de la compañía o empresa ver que algo no está ubicado o no funciona correctamente?; si esta persona detecta una situación incorrecta itiene las indicaciones necesarias y suficientes para actuar en consecuencia sin depender de otras personas? Fundación Vasca para la Excelencia - Euskalit (sf), refiere que definitivamente si las respuestas son positivas, implica que se ha estandarizado nuestros procesos y que estamos cumpliendo las 35 anteriores. En pocas palabras Seiketsu, consiste en distinguir fácilmente una situación normal de otra anormal, mediante normas sencillas y visibles para todos.

\section{SHITSUKE}

La disciplina es fundamental y básica para lograr el éxito en cualquier institución. Shitsuke, significa disciplinar e implica entrenar a los colaboradores para que apliquen con disciplina las buenas prácticas de orden y limpieza; hacer un hábito las primeras 45 mediante la capacitación, motivación y disciplina con el fin de lograr el éxito de las actividades productivas.

Si nos centramos en esta pregunta: ¿Está su lugar de trabajo mejor organizado, Más lim- 
pio y ordenado que hace un año? Entonces se puede establecer la relación que implica trabajar con disciplina y esto hacerlo un hábito. Disciplina y hábito consiste en trabajar permanentemente de acuerdo con las normas establecidas, donde todos los colaboradores asumen este compromiso para mantener y mejorar el nivel de Organización, Orden y Limpieza en las labores cotidianas del trabajo.

Hablar de disciplina no es hablar de obligación, menos de una imposición de reglas, normas o procedimientos. Se puede resumir que actuar con disciplina es actuar acorde a lo establecido por convicción propia. La acción repetida de las actividades siguiendo las normas y procedimientos genera el hábito, la practica constante de estos los hace más fuertes. Sin disciplina no hay hábitos y si esto no se logra pues todo el esfuerzo realizado en la implementación de las primeras $4 \mathrm{~S}$ pues habrá sido en vano. En pocas palabras disciplina significa convertir en hábito el mantenimiento de los procedimientos correctos (TGP Hoshin (sf).

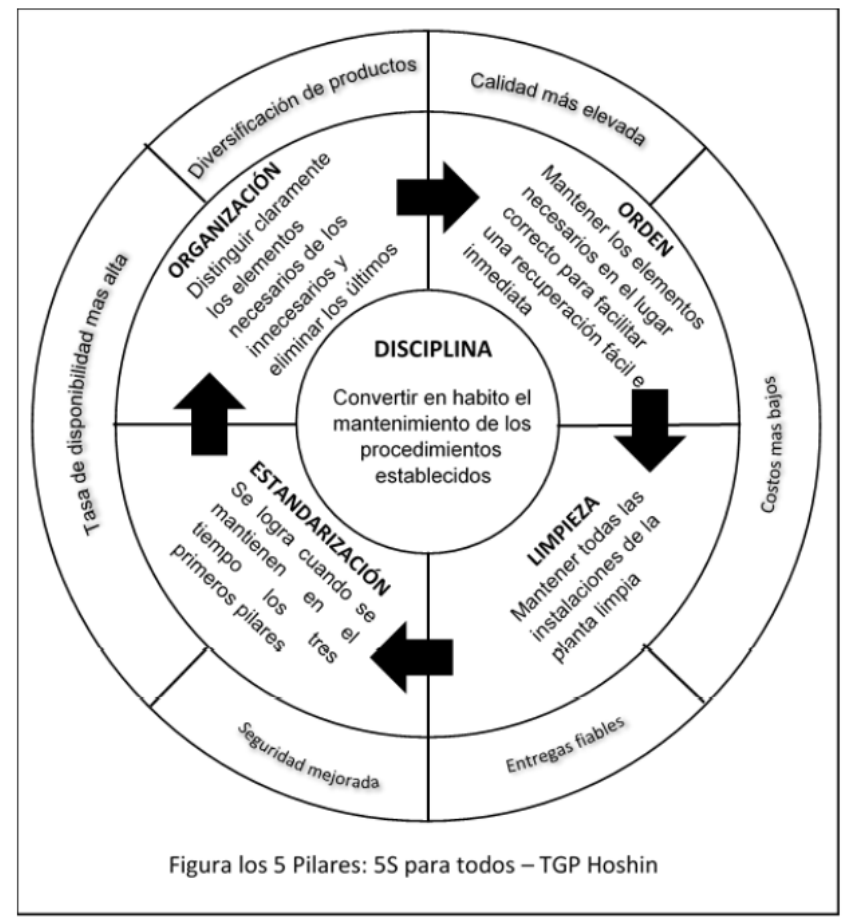

\section{LA IMPLEMENTACIÓN DE LAS 5 S}

La implementación de las 55 se da en una etapa para cada una de las "S", ya que cada una implica una mejora en el proceso; para cada una de ellas se debe llevar a cabo los mismos pasos de implementación. Para su implementación cada grupo de trabajo determina las necesidades y tareas que deben realizar cada una de las "S".

El primer paso para la implementación es la reunión de inicio a la implementación, la misma que busca informar sus objetivos, capacitar a los colaboradores, diagnosticar la situación inicial y establecer las oportunidades de mejora. Este paso es fundamental y debe estar dirigido por el responsable líder de la implementación acompañado de un representante de la alta dirección. En este paso quedan establecidos los lineamientos a seguir en la segunda etapa.

El segundo paso es el proceso de implementación propiamente dicho, en este paso después de haber hecho el diagnóstico y establecido las oportunidades de mejora en el primer paso, se organizan los equipos de trabajo que 
se encargarán de realizar las acciones para la mejora del proceso. Es el líder de la implementación, el llamado a dar seguimiento a las diferentes actividades programadas. Las herramientas de gestión adecuadas según las actividades y el registro fotográfico (Gestión Visual) son fundamentales en esta etapa. El registro fotográfico del antes, es fundamental para tener una guía de cómo no debemos estar; además, de ser un indicador de cuando nos estemos desviando del objetivo.

La tercera etapa es la etapa de Evaluación y parte del control y las inspecciones. Establecer indicadores es fundamental para la evaluación: debemos recordar: "Lo que no se mide, no se puede mejorar" (Peter Druker), por lo que es fundamental medir el nivel de implementación de las 5S. Hoy en día, mediante hojas de cálculo es fácil llevar un control del nivel de implementación para cada una de las 5 S. La evaluación periódica utilizando instrumentos y formatos de registro sencillos, establecidos según la necesidad, sirven para evaluar el nivel de implementación y tiene como base la información levantada durante las inspecciones de rutina para las $5 \mathrm{~S}$.

El cuarto paso de la implementación es la difusión. Este paso es muy importante ya que, al ser un proceso de mejora, difundir los resultados ayuda a reconocer el trabajo de los colaboradores y motivar a los mismos a continuar con el proceso. El uso de murales, la implementación de un buzón de sugerencias - los carteles de motivación, como el cartel del trabajador del mes son alternativas muy buenas para este paso.

Las $5 \mathrm{~S}$ son base fundamental de otras técnicas de gestión y mejora continua como el Lean Manufacturing y el TPM (Mantenimiento Productivo Total) de hecho estas metodologías tienen como pilares la filosofía de las 55 .

Rajadell C. M.\& Sánchez G. J.L, (2010) manifiestan en su libro Lean Manufacturing, la evidencia de una Necesidad que el Lean Ma- nufacturing, tiene por objetivo la eliminación del despilfarro, mediante la utilización de una colección de herramientas (TPM, 5S, SMED, kanban, kaizen, heijunka, jidoka, etc.), que se desarrollaron fundamentalmente en Japón. Los pilares del Lean Manufacturing son: la filosofía de la mejora continua, el control total de la calidad, la eliminación del despilfarro, el aprovechamiento de todo el potencial a lo largo de la cadena de valor y la participación de los colaboradores.

Renovetec (2009) en su revista Colección Mantenimiento Industrial, Técnicas Avanzadas de Gestión del Mantenimiento en la Industria, manifiesta: EI TPM al igual que la filosofía Kaizen nace en Japón como una técnica de la Japan Institute of Plant Maintenance (JIPM), destinado a lograr la eliminación de las Ilamadas <seis grandes pérdidas> del proceso productivo, y con el objetivo de facilitar la implantación de la forma de trabajo "Just in Time" o "justo a tiempo" otra de estas técnicas que forman parte de un conglomerado de estrategias del Lean Manufacturing (Fabricación ajustada). TPM es una filosofía de mantenimiento cuyo objetivo es eliminar las pérdidas de los sistemas productivos debido al estado de los equipos, o en otras palabras, aumentar la disponibilidad de estos para asegurar los más altos niveles de productividad. Lograr mantener cero averías, cero tiempos muertos, cero defectos como consecuencia al mal estado de los equipos y las pérdidas de rendimiento o de capacidad productiva, ya que son los objetivos deseados por el TPM y las 5S, es su herramienta más efectiva para alcanzarlo.

Son la 5 S para el mundo empresarial e industrial emergente una filosofía fundamental e indispensable en la búsqueda de la ansiada excelencia en la calidad.

\section{CONCLUSIONES}

Para muchos implementar las $5 \mathrm{~S}$ significa incrementar actividades de trabajo a las propias de la actividad laboral de cada uno de los co- 
laboradores; sin embargo, por la misma experiencia de empresas donde ya se aplica y funciona perfectamente bien esta metodología se podría inferir que mejora el trabajo. Reduce las pérdidas de tiempo en los procesos y permite tomar acción inmediata frente a las situaciones adversas que se pueden presentar.

Las S logran resultados de mejora significativos en los procesos productivos. Optimiza la calidad de los productos o servicios, ya que disminuye el error o fallas en el proceso; mejora la productividad, ya que elimina desperdicios tanto de tiempo, materias primas, recursos en general; mejora la seguridad, ambientes ordenados son ambientes seguros, procesos estandarizados reducen considerablemente la probabilidad de accidentes. Un factor importante que se logra con la implementación de las 55 es el clima laboral, es decir, mejora considerablemente el ambiente de trabajo, favorece el desarrollo de la comunicación efectiva entre los colaboradores, incrementa y desarrolla su creatividad; así mismo, permite el crecimiento profesional.

Freyre (2017), en su tesis "Relación de la Metodología 5 "S" y los Procesos Operativos del Almacén de Distribuidoras en Lima Metropolitana" concluyó que la estandarización de procesos operativos influirá en la eficiencia de procedimientos operativos del almacén de distribuidoras, mostrando que existe una fuerte relación entre la estandarización de procesos operativos y la mejora de la eficiencia de procedimientos; confirmando que al estandarizar los procesos se simplifican las tareas creando eficiencia en los procedimientos.

Definitivamente cuando un proceso de mejora es estandarizado aporta positivamente a levantar los indicadores de productividad y como consecuencia la rentabilidad de un proceso.

Por otra parte, Chilón, Xiomara; Esquivel, Lourdes; Tamay Walter (2017), logran demostrar como la implementación de las 55 produce un incremento significativo en la productividad de la empresa. Mediante un diseño experimental, analizando los datos y valores de productividad de los meses de febrero a julio del año 2016; a través del diagnóstico situacional, determinaron inicialmente que el 100\% de los trabajadores no tenían conocimiento de las 5S; además, que no existía un plan sistemático de organización y limpieza de la línea de producción. Utilizando formatos específicos de evaluación de los pasos de la metodología 5S pudieron determinar en un estado inicial que existían en el proceso productivo $55 \%$ y $45 \%$ de materiales necesarios e innecesarios, respectivamente. Otra de las situaciones significativas encontradas es que el $60 \%$ de las áreas están poco estandarizadas. La evaluación inicial de la implementación reportó un cumplimiento del $66.18 \%$. Luego de la implementación de las 55 , se pudo determinar según los registros de producción diarios un incremento en los niveles de producción de $103.41 \mathrm{~L}$ a 133.39L es decir un incremento del $29 \%$. Inicialmente, se puede decir que el desconocimiento de la metodología de las 5S, a pesar de ser muy antigua, aún en nuestro entorno laboral es desconocida; sin embargo, está demostrado que con esta se logran incrementos positivos muy significativos en los procesos de mejora continua.

En cuanto a la organización del trabajo, Fuentes (2017) en su tesis "Implementación de la Metodología 5 "S" para reducir los tiempos en la ubicación de documentos en el Área de Aseguramiento y Control de la Calidad de una entidad bancaria", determinó que con la aplicación de la Metodología 5S, se logró reducir los tiempos de búsqueda de documentación hasta un $99 \%$, en los casos más críticos y un 85 $\%$ en los casos menos críticos; ello debido a que, se dio prioridad de ubicación a los documentos con mayor importancia y utilidad. Este mismo efecto se puede lograr si se aplica por ejemplo en el área de mantenimiento en una planta industrial. El tener las herramientas y repuestos de forma ordenada reducirá los tiempos de intervención, en caso de reali- 
zarse una reparación o corrección de un desperfecto. Las paradas de planta son el principal factor que afecta la productividad en una línea de producción por lo que lograr reducir tiempos en la intervención y reparación incrementaría considerablemente la disponibilidad de la misma.

Otro de los aspectos más importantes del impacto positivo de las $5 S$ en las empresas la mejora de las relaciones laborales, lo evidenció. Oré (2017), en su tesis "Implementación de la metodología 55 en el área de Logística Recepción de la empresa Gloria S.A." determinando que después de la implementación de las $5 \mathrm{~S}$ se logró mejorar el clima laboral, aumentando en más del 50\% el nivel de motivación, comunicación, estructura y liderazgo que presentaba el área en estudio. Esto se vio reflejado en los resultados del cuestionario diagnóstico realizado para tal fin.

En este orden de ideas la Fundación Vasca para la Excelencia (Euskalit), concluye que después de implementada las 55 se logra un considerable tiempo y eficiencia los procesos y operaciones, lo que demandan ahorros significativos para la empresa.

La comunicación sobre los logros alcanzados con la implementación y práctica de las $5 S$ son muy importantes, para mantener en el tiempo esta estrategia; el implementar murales o posters de los avances y logros, mantiene motivado al personal y valoriza el trabajo de los mismos. Una imagen muchas veces vale más que mil palabras; la muestra de fotografías del antes y después de la implementación de las $5 \mathrm{~S}$ son herramientas de alto impacto, para promover esta estrategia ya que indican, no solo los logros alcanzados, sino el nivel de avance en la implementación, lo que permitirá hacer seguimiento, control y posterior mejora del proceso.

\section{REFERENCIAS BIBLIOGRÁFICAS}

Almonte, S.K. (2011). Kaizen y las 5 S.[Blog: Escuela de Organización Industria]. Recuperado de: https://www.eoi.es/blogs/karlasugeilyalmonte/2011/12/16/kaizen-y-las-5s/

Carvalho de Souza, B., Alcantara, G., Parreira, P., Et al (2018). Implementación del programa $5 S$ a través de la metodología DMAIC. Brazilian Journal of Development. ISSN: 25258761. Recuperado de: https://www.brazilianjournals.com/index.php/BRJD/article/ view/245/204

Centro de Innovación Tecnológica de la Madera (20085). Método 5 S Aplicado a la Industria de la Madera y el Mueble. Recuperado de: https://cdn.www.gob.pe/uploads/document/file/571591/01._5s.pdf

Chiavenato. (2008). Gestión del Talento Humano. Mc Graw Hill. 3a. edición.

Chilón, X.; Esquivel, L.; Tamay, W (2017). Implementación de las 5 s para incrementar la productividad en una planta embotelladora de agua. Ingnofis. Recuperado de: http://revistas.ucv.edu.pe/index.php/INGnosis/article/ view/2028/1718

Cruz, J (2010). Manual para la Implementación de las 5 "S" https://issuu.com/victore.cardozodelgado/docs/manual_para_la_implementaci__n_sost

Custer, D. (1962). La Mente en las relaciones Humanas. España: C.E.C.S.A.

Freyre, R.K. (2017). Relación de la Metodología $5 S$ y los Procesos Operativos del Almacén de Distribuidoras en Lima Metropolitana. (Tesis para Título Profesional). Universidad san Ignacio de Loyola. Perú

Fuentes L, K.D. (2017). Implementación de la metodología 5S para reducir los tiempos en la ubicación de documentos en el área de Aseguramiento y Control de la Calidad de una entidad bancaria (Tesis para Título Profesional). Universidad Nacional Mayor de San Marcos. Perú 
Fundación Vasca para la Excelencia - Euskalit. Metodología de Las 5 "S" Mayor Productividad Mejor Lugar de Trabajo. Recuperado de: http://www.euskalit.net/pdf/folleto2.pdf

Gomes, L., (2006). Reavaliação e melhoria dos processos de beneficiamento de não tecidos com base em reclamações de clientes. Revista. $F A E$, Curitiba.

Mateos, J. (2010). 5S en la Industria Alimentaria. Recuperado de https://es.scribd.com/ document/461135019/5S-en-la-Industria-Alimentaria-Javier-Mateos-Escamilla

Ore R., K.L. (2016). Implementación de la metodología $5 S$ en el área de logística recepción de la empresa Gloria S.A. (Tesis para Título Profesional). Universidad Nacional Mayor de San Marcos. Perú.

Poma A., S.J. (2017). Propuesta de implementación de la Metodología de las 55 para la mejora de la gestión del almacén de suministros en la Empresa Molitalia S.A. Sede los olivos Lima, 2017. (Tesis para Título Profesional). Universidad Nacional de Ingeniería. Perú.

Rajadell C. M., Sánchez G. J.L, (2010). Lean Manufacturing, la evidencia de una Necesidad. España: Ediciones Díaz de Santos.

Renovetec (2009). Mantenimiento Industrial, Técnicas Avanzadas de Gestión del Mantenimiento en la Industria, Vol 6. Recuperado de https://www.freelibros.me/libros/ingenieria-del-mantenimiento-tecnicas-avanzadas-de-gestion-del-mantenimiento-en-la-industria

Rojas R., M.S. (2017). Propuesta de una Metodología para la Implementación de Las 5 "S" en una Empresa Productora de Alimentos. (Tesis para Título Profesional) Universidad Técnica Federico Santa María. Chile. Recuperado de: https://repositorio.usm.cl/bitstream/ handle/11673/23258/3560900232501UTFSM. pdf? sequence $=1$ \&isAllowed $=y$.
TGP Hoshin (sf). 5S para todos. Recuperado de https://es.scribd.com/document/390802786/5S-Para-Todos-pdf

\section{CORRESPONDENCIA:}

Mag. Lucia Pantoja-Tirado

luciapantoja@unat.edu.pe 